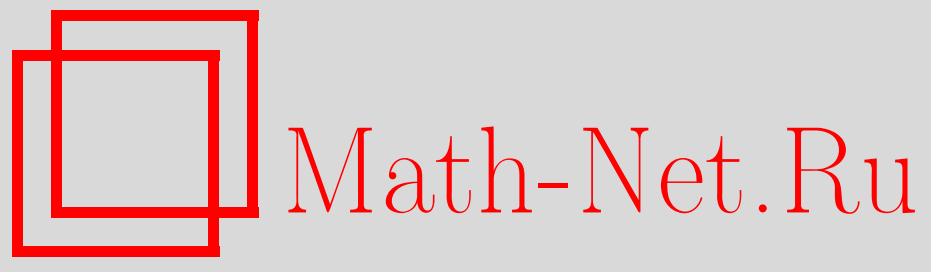

А. Р. Чехлов, О прямой сумме неприводимых групп, $M a-$ тем. заметки, 2015, том 97, выпуск 5, 798-800

DOI: https://doi.org/10.4213/mzm10657

Использование Общероссийского математического портала Math-Net.Ru подразумевает, что вы прочитали и согласны с пользовательским соглашением http://www . mathnet.ru/rus/agreement

Параметры загрузки:

IP: 54.80 .73 .141

26 апреля 2023 г., 10:14:10

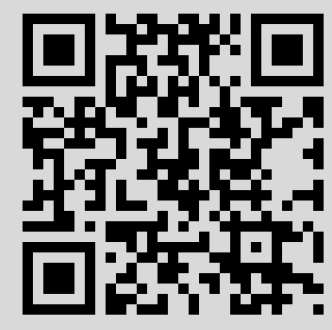




\section{О прямой сумме неприводимых групп}

\section{А. Р. Чехлов}

Все группы в заметке предполагаются абелевыми. Через $E(A)$ обозначается кольцо эндоморфизмов группы $A$. Напомним, что подгруппа $G$ группы $A$ называется чистой, если $G \cap n A=n G$ для всех целых чисел $n$. Группа называется неприводимой, если она не имеет собственных чистых вполне инвариантных подгрупп. Неприводимые группы изучались в [1], [2] и в др. работах; подробно с приведением литературы они рассматриваются в [3]. Поскольку ядро всякого центрального эндоморфизма вполне инвариантно, все ненулевые центральные эндоморфизмы неприводимой группы без кручения являются мономорфизмами.

Группу без кручения $A$ назовем qft-группой (квази вполне транзитивной), если для любых элементов $0 \neq a, b \in A$ условие на типы $t(a) \leqslant t(b)$ влечет существование $f \in E(A)$ и натурального числа $k$ со свойством $f(a)=k b$. Если для любых $0 \neq a, b \in A$ условие на характеристики $\chi(a) \leqslant \chi(b)$ (соответственно, $\chi(a)=\chi(b)$ ) влечет существование $f \in E(A)$ (соответственно, автоморфизма $f$ группы $A$ ) со свойством $f(a)=b$, то группа $A$ называется вполне транзитивной (соответственно, транзитивной). Вполне транзитивным и близким к ним классам групп без кручения (транзитивным, сильно однородным и др.) посвящена глава VII книги [3]. Легко видеть, что группа без кручения неприводима тогда и только тогда, когда она является однородной qft-группой; qft-группы можно рассматривать как обобщение неприводимых групп и вполне транзитивных групп без кручения.

Напомним, что кольцо $R$ с единицей называется Е-кольщом [4], если $\operatorname{Hom}_{\mathbb{Z}}(R, R)=$ $\operatorname{Hom}_{R}(R, R)$, где $\mathbb{Z}$ - кольцо целых чисел. Будем говорить, что кольцо $S$ обладает свойcтвом $(*)$, если его аддитивная группа $S^{+}$есть редуцированная группа без кручения и факторкольцо $S / p S$ не имеет делителей нуля для каждого простого числа $p$ такого, что $p S \neq S$; согласно [3; лемма 44.6] в группе $S^{+}$такого кольца $S$ для любых $a, b \in S^{+}$имеет место равенство $\chi(a b)=\chi(a)+\chi(b)$.

Лемма 1. Пусть $R$ есть Е-кольцо со свойством (*). Тогда эквивалентны следующие условия:

(1) группа $R^{+}$неприводима;

(2) каждый элемент кольца $R$ есть целое кратное обратимого;

(3) $R^{+}$- однородная вполне транзитивная группа.

ДоказАтельство. $(1) \Rightarrow(2)$ Так как группа $R^{+}$неприводима, она однородна, и поскольку характеристика $\chi(1)$ наименьшая, то тип группы $R^{+}$идемпотентный. Пусть $I=$ $x R$ - главный идеал, $x=n x_{0}$, где $\chi\left(x_{0}\right)=\chi(1), J=x_{0} R$. Если $y=x_{0} z \in J$, где $z \in R$, то

$$
\chi(y)=\chi\left(x_{0}\right)+\chi(z)=\chi(z),
$$

так как характеристика $\chi\left(x_{0}\right)$ состоит только из 0 и $\infty$ и символ $\infty$ у $\chi\left(x_{0}\right)$ и $\chi(z)$ встречается на одних и тех же местах. Поэтому если $p^{k} t=y$, то $z \in p^{k} R$ и $y \in p^{k} J$, т.е. $J^{+}-$чистая вполне инвариантная (так как $R-$ Е-кольцо) подгруппа в $R^{+}$. Следовательно, $J=R$; в частности, элемент $x_{0}$ обратим.

$(2) \Rightarrow(3)$ Так как каждый элемент из $R$ является целым кратным обратимого, то группа $R^{+}$однородна идемпотентного типа. Пусть теперь $0 \neq a, b \in R$ и $\chi(b) \geqslant \chi(a)$ в группе $R^{+}$, и пусть $n a_{0}=a$, где $a_{0}$ обратим. Тогда $n b_{0}=b$ для некоторого $b_{0} \in R$ и $b_{0} a_{0}^{-1}(a)=b$.

Импликация (3) $\Rightarrow(1)$ очевидна.

DOI: $10.4213 / \mathrm{mzm} 10657$ 
Кольцо $R$ с условием (2) леммы 1 называется сильно однородным, каждый его идеал является главным и имеет вид $n R$, где $n$ - некоторое целое число [3; $\S 19]$. Каждое из утверждений леммы эквивалентно тому, что $R^{+}$является однородной транзитивной группой. Напомним, что группа $A$ называется эндоконечной [3; определение 8.3], если она является конечно порожденным $E(A)$-модулем.

Предложение 1. Счетная неприводимая эндоконечная группа без кручения $A$, у которой центр $C$ кольца $E(A)$ есть кольио главных идеалов со свойством (*), является вполне транзитивной и транзитивной группой.

ДоказАтельство. Согласно [3; теорема 8.7] $C$ является Е-кольцом, а по следствию 8.6 из [3] $A$ есть свободный $C$-модуль. Как прямое слагаемое группа $C^{+}$также неприводима; значит, кольцо $C$ удовлетворяет условиям леммы 1 ; осталось сослаться на следствие 40.5 из [3].

СледствиЕ 1. Неприводимая эндоконечная группа без кручения $A$ конечного ранга, у которой центр $C$ кольца $E(A)$ есть кольцо со свойством $(*)$, является вполне транзитивной и транзитивной группой.

Доказательство. Согласно следствию 8.8 из [3] $C$ есть Е-кольцо и $A$ квазиизоморфна $C^{n}$ для некоторого $n$. Как квазислагаемое в $A$ группа $C^{+}$также является неприводимой. Осталось сослаться на лемму 1 и предложение 1.

Tеорема 1. Пусть $A_{i} \neq 0, i \in I,-$ неприводимые группы без кручения $u|I| \geqslant 2$. Группа $A=\bigoplus_{i \in I} A_{i}$ является неприводимой тогда и только тогда, когда $\operatorname{Hom}\left(A_{i}, A_{j}\right) \neq 0$ для каждой пары $i, j \in I$.

ДоказАтельство. Необходимость. Допустим, что $\varphi(a)=0$ для некоторого $0 \neq a \in A_{i}$ и для каждого $\varphi \in \operatorname{Hom}\left(A_{i}, A_{j}\right)$. Положим $B=\bigoplus_{k \in I \backslash\{j\}} A_{k}$ и $C=A_{j}$. Тогда

$$
a \in H=\bigcap_{f \in \operatorname{Hom}(B, C)} \operatorname{ker} f
$$

$H$ является чистой вполне инвариантной подгруппой группы $A$. Имеем

$$
H=\bigoplus_{i \in I}\left(H \cap A_{i}\right)
$$

где $H \cap A_{i}$ - чистая вполне инвариантная подгруппа в $A_{i}$. Следовательно, $H \cap A_{i}=A_{i}$, если $H \cap A_{i} \neq 0$. Значит, $H$ является вполне инвариантным прямым слагаемым группы $A$, что противоречит ее неприводимости.

Достаточность. Если $H \neq 0$ - чистая вполне инвариантная подгруппа группы $A$, то $H \cap A_{i} \neq 0$ (значит, $\left.H \cap A_{i}=A_{i}\right)$ хотя бы для одного $i \in I$. Условие $\operatorname{Hom}\left(A_{i}, A_{j}\right) \neq 0$ для каждой пары $i, j \in I$ и qft-транзитивность групп $A_{i}$ влечет, что $H \cap A_{i} \neq 0$ (значит, $\left.H \cap A_{i}=A_{i}\right)$ для каждого $i \in I$. Откуда $H=A$.

Будем говорить, что упорядоченная пара $(B, G)$ групп без кручения $B$ и $G$ удовлетворяет правому qft-условию, если для любых $0 \neq b \in B, 0 \neq g \in G$ таких, что $t(b) \leqslant t(g)$ найдутся $f \in \operatorname{Hom}(B, G)$ и натуральное $k$ со свойством $f(b)=k g$.

ПреДЛОЖенИЕ 2. Пусть $A_{i} \neq 0(i \in I)$ - неприводимые группы без кручения $u|I| \geqslant \aleph_{0}$. Группа $A=\prod_{i \in I} A_{i}$ является неприводимой тогда и толъко тогда, когда $\operatorname{Hom}\left(A_{i}, A_{j}\right) \neq 0$ для каждой пары $i, j \in I$, все $A_{i}$ - однородные группы одного и того же идемпотентного типа и хотя бъ для одного $i \in I$ пара $\left(A_{i}, G_{i}\right)$, где $\left(G_{i}=\prod_{j \in I \backslash\{i\}} A_{j}\right)$, удовлетворяет правому qft-условию. 
Доказательство. Если группа $A$ неприводима, то она однородна, поэтому все $A_{i}$ имеют один и тот же идемпотентный тип [5; доказательство леммы 96.4]; необходимость оставшихся утверждений очевидна.

Достаточность. Пусть $H \neq 0$ - чистая вполне инвариантная подгруппа группы $A$. Тогда $\pi_{i}(H) \neq 0$ хотя бы для одной проекции $\pi_{i}: A \rightarrow A_{i}$ и, значит, $H \cap A_{i} \neq 0$. Как чистая вполне инвариантная подгруппа неприводимой группы $H \cap A_{i}$ совпадает с $A_{i}$. Поскольку $\operatorname{Hom}\left(A_{i}, A_{j}\right) \neq 0$ для каждой пары $i, j \in I$, отсюда следует, что

$$
\bigoplus_{i \in I} A_{i} \subseteq A
$$

а правое qft-условие пары $\left(A_{i}, G_{i}\right)$ влечет существенность подгруппы $H \cap G_{i}$ в $G_{i}$; ее чистота дает равенство $H \cap G_{i}=G_{i}$. Откуда $H=A$.

\section{СПИСОК ЦИТИРОВАННОЙ ЛИТЕРАТУРЫ}

[1] J. D. Reid, Topics in Abelian Groups, Scott, Foresman and Co., Chicago, IL, 1963, 51-58. [2] П. А. Крылов, Абелевы группы и модули, 1986, № 4, 73-100. [3] П. А. Крылов, А. В. Михалев, А. А. Туганбаев, Абелевы группь и их кольца эндоморфизмов, Факториал Пресс, М., 2006. [4] P. Schultz, J. Austral. Math. Soc., 15 (1973), 60-69. [5] Л. Фукс, Бесконечные абелевы группы, Т. 2, Мир, М., 1977.

\section{А. Р. Чехлов}

Томский государственный университет

E-mail: cheklov@math.tsu.ru
Поступило

01.12 .2014 